\title{
Efectividad de las vitaminas C y E como tratamiento adyuvante a la terapia triple estándar para Helicobacter pylori en una cohorte de la Amazonía peruana
}

\section{Effectiveness of vitamins C and E adjuvant to standard triple therapy for Helicobacter pylori in a cohort from the Peruvian Amazon}

\author{
Wildor Samir Cubas, ${ }^{1 *}$ Rómulo Reyes Cahuila, ${ }^{2}$ Heriberto Arévalo Ramírez, ${ }^{3}$ Antonio M. Quispe. ${ }^{4}$
}

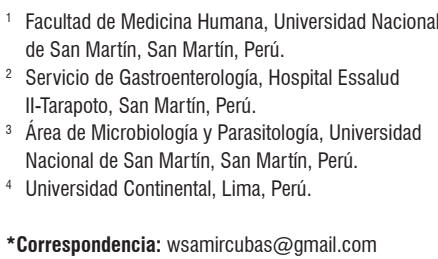

\begin{abstract}
Resumen
Introducción y objetivos: la terapia con vitaminas C y E ha sido propuesta como adyuvante a la terapia triple estándar (TTE) con el fin de incrementar la tasa de erradicación del Helicobacter pylori (H. pylori). En este estudio probamos esta hipótesis en una cohorte de pacientes de la Amazonía peruana. Materiales y métodos: retrospectivamente, evaluamos una cohorte de 50 pacientes infectados con $\mathrm{H}$. pylori del Hospital de Tarapoto en el período comprendido entre julio-diciembre de 2016; de estos, 25 fueron tratados con TTE (amoxicilina $1 \mathrm{~g}$, claritromicina $500 \mathrm{mg}$ y omeprazol $20 \mathrm{mg}$, dos veces al día por 14 días) en adyuvancia con las vitaminas $C$ y $E$, y 25 fueron tomados al azar (1:1), quienes solo recibieron TTE. Se estimó y comparó la efectividad de ambos tratamientos utilizando un modelo regresión general lineal con familia Poisson y link log, teniendo como desenlace de interés la erradicación del $H$. pylori confirmada por histopatología. Resultados: al comparar la cohorte de expuestos y con los no expuestos, no se encontraron diferencias significativas en sus características basales, incluyendo edad (38 \pm 11 frente a $36 \pm 10$ años), género masculino (65\% frente a $63 \%$ ), síntomas y diagnóstico histopatológico. Al comparar la efectividad de ambos tratamientos, se encontró un incrementó no significativo en las tasas de erradicación del 9,5 \% (91 \% frente a 82 \%, razón de tasas de incidencia = 1,11; intervalo de confianza [IC] $95 \%: 0,92$ a 1,36). Conclusiones: la terapia adyuvante con vitaminas $\mathrm{C}$ y E podría ayudar a incrementar la efectividad de la TTE para $\mathrm{H}$. pylori en pacientes de la Amazonía peruana, aunque se requiere confirmar esta hipótesis en un ensayo clínico.
\end{abstract}

Palabras clave

Ácido ascórbico, vitamina E, Helicobacter pylori, Perú.

\begin{abstract}
Introduction and Objectives: Adjuvant therapy with vitamins $C$ and $E$ has been proposed to increase standard triple therapy's Helicobacter pylori eradication rate. This study tested this hypothesis in a cohort of patients from the Peruvian Amazon. Material and Methods: We retrospectively evaluated a cohort of 50 patients at Tarapoto Hospital who were treated for $\mathrm{H}$. pylori infections from July to December 2016. Of these, 25 were randomly selected to receive standard triple therapy of $1 \mathrm{~g}$ amoxicillin, $500 \mathrm{mg}$ clarithromycin and $20 \mathrm{mg}$ omeprazole twice a day for 14 days plus adjuvant vitamins $C$ and $E$. The other 25 only received standard triple therapy. The effectiveness of both treatments was estimated and compared using a general linear regression model using the Poisson family of distributions and log link with $\mathrm{H}$. pylori eradication confirmed by histopathology as the outcome of interest. Results: A comparison of the two groups found no significant differences in their baseline symptoms, histopathological diagnoses, ages (38 \pm 11 years vs. $36 \pm 10$ years) or genders ( $65 \%$ male vs. $63 \%$ male). A comparison of the effectiveness both treatments found a non-significant increase in eradication rates of $9.5 \%(91 \%$ vs. $82 \%$, incidence rate ratio $=1.11 ; 95 \%$ confidence interval: 0.92 to 1.36). Conclusions: Adjuvant therapy with vitamins $C$ and $E$ may help increase the effectiveness of standard triple therapy for $\mathrm{H}$. pylori in patients in the Peruvian Amazon, although this hypothesis needs to be confirmed in a clinical trial.
\end{abstract}

Keywords

Ascorbic acid; Vitamin E; drug therapy; Helicobacter pylori; Peru (source: Decs BIREME). 


\section{INTRODUCCIÓN}

La infección por $H$. pylori es una de las infecciones bacterianas más frecuentes en adultos a nivel mundial (1). En países en desarrollo, se estima que tiene una prevalencia por encima del $70 \%$, mientras en países desarrollados es cercana al $35 \%$ (2). Esta bacteria coloniza el estómago y suele producir síntomas en el $32 \%$ de los casos, siendo los más frecuentes el dolor abdominal, la regurgitación, la pirosis, las náuseas y la hiporexia (3). En la actualidad, la Organización Mundial de la Salud (OMS) ha catalogado al $H$. pylori como un agente carcinógeno tipo 1, debido a su estrecha relación con el cáncer gástrico (4). Por esta razón, se recomienda su inmediata erradicación al momento del diagnóstico, ya que es un microorganismo de alta patogenicidad (5).

En la actualidad, el tratamiento de primera línea para erradicar el H. pylori es la terapia triple estándar (TTE), la cual consiste en la administración de dos antibióticos (amoxicilina $2 \mathrm{~g}$ dos veces al día y claritromicina $500 \mathrm{mg}$ dos veces al día) y un inhibidor de bomba de protones (omeprazol 20 mg dos veces al día) de forma diaria por dos semanas (5). Hasta la década pasada, con esta terapia, en el mejor de los casos, se alcanzaban tasas de erradicación del $H$. pylori del $77-82 \%(6,7)$; desde entonces, se han propuesto diferentes modificaciones a la TTE con el objeto de incrementar estas tasas, entre las que podemos destacar la terapia cuádruple, la secuencial, la híbrida y la adyuvante con vitaminas $\mathrm{C}$ y E (8). Precisamente, esta última ha generado gran expectativa al tratarse de una terapia relativamente inocua y accesible, y porque, según algunos reportes, puede contribuir a elevar las tasas de erradicación por encima del 90 \% (9). En el caso de la vitamina $C$, se ha demostrado que su efecto adyuvante se debe a su capacidad de inhibir la formación de compuestos $\mathrm{N}$-nitroso y metabolitos reactivos de oxígeno en la mucosa gástrica (10), ambos considerados vitales para el crecimiento y la carcinogenicidad del H. pylori (11). Por su parte, el efecto adyuvante de la vitamina $\mathrm{E}$ se debe a su capacidad para inhibir la peroxidación de los lípidos y el estrés oxidativo a nivel del microambiente creado por esta bacteria en la mucosa gástrica (12), también considerados factores esenciales para el crecimiento y patogenia del H. pylori (13).

Los primeros estudios que evaluaron los efectos de la terapia adyuvante con vitaminas $\mathrm{C}$ y $\mathrm{E}$ arrojaron resultados poco favorables (14). De hecho, el primer metaanálisis publicado por Li en el 2011 planteó que las evidencias disponibles hasta ese momento para recomendar dicha terapia carecían de hallazgos significativos (riesgo relativo $=0,93$; intervalo de confianza (IC) del $95 \%$ : 0,56-1,53) (15). Sin embargo, los nuevos estudios realizados muestran resultados satisfactorios y significativos, y le atribuyen a la terapia adyuvante con vitaminas $\mathrm{C}$ y $\mathrm{E}$ tasas de erradicación que sobrepasan el $20 \%$ de diferencia frente a la terapia triple estándar (16). Estos valores podrían ser incluso mayores en el caso de pacientes con bajos niveles de capacidad antioxidante y anemia ferropénica, según los últimos reportes publicados $(17,18)$. Esto último es muy importante debido que en el Perú, la prevalencia de anemia ferropénica supera el $40 \%$ (19), y en las regiones de la selva peruana, incluyendo el departamento de San Martín, estos valores oscilan alrededor del $24 \%$ (20). En la mayoría de casos, esta patología carencial está asociada con la mala absorción del hierro en presencia de bajas concentraciones de vitamina $C$ (21); por esta razón, el presente estudio intenta determinar la efectividad atribuible al uso de antioxidantes (vitaminas C y E) como tratamiento adyuvante a la TTE para $H$. pylori en una cohorte de pacientes de la Amazonía peruana.

\section{MATERIALES Y MÉTODOS}

\section{Diseño del estudio}

Se realizó un estudio de cohorte retrospectivo, en el distrito de Tarapoto, provincia de San Martín $\left(6^{\circ} 29^{\prime} 00^{\prime \prime} \mathrm{S}, 76^{\circ} 22^{\prime} 00^{\prime \prime} \mathrm{O}\right.$, población $\sim 118,000$ ), departamento de San Martín, en la región del nororiente del Perú. Se evaluó a toda la cohorte de pacientes con infección por $H$. pylori diagnosticados en el Hospital del Seguro Social (EsSalud) de Tarapoto entre los meses de julio a diciembre del 2016, quienes recibieron como tratamiento la TTE en adyuvancia con las vitaminas C y E (expuestos). Con la finalidad de estimar el efecto atribuible del tratamiento adyuvante con las vitaminas $\mathrm{C}$ y E, se comparó a la cohorte de expuestos con una cohorte de no expuestos. Para ello, se tomó una muestra (1:1) al azar (por muestreo aleatorio simple) del total de pacientes que durante el mismo período de tiempo fueron diagnosticados con infección por H. pylori, tratados con la TTE y no fueron expuestos a la terapia adyuvante con vitaminas $\mathrm{C}$ y E. Para comparar la efectividad del tratamiento, ambas cohortes (expuestos y no expuestos) fueron seguidas retrospectivamente para medir y comparar la tasa de erradicación del H. pylori. Con el objeto de evitar sesgo de información, se planteó a priori que para declarar la erradicación del H. pylori, esta debía ser confirmada por histopatología posendoscopía digestiva alta al término del tratamiento.

\section{Población y muestra}

La cohorte de expuestos estuvo constituida por todos los casos de pacientes que cumplieron con los siguientes criterios de inclusión: adultos con edades comprendidas entre los 18-60 años, sintomáticos (dolor abdominal, regurgitación, pirosis, náuseas y/o hiporexia), con diagnóstico histopatológico de infección por $H$. pylori, que recibieron como tratamiento la TTE en adyuvancia con vitaminas $\mathrm{C}$ 
y E. Por su parte, la cohorte de no expuestos fue tomada al azar (muestra 1:1) entre todos los pacientes con diagnóstico histopatológico de infección por $H$. pylori, que solo recibieron como tratamiento la TTE. En ambas muestras se excluyeron a todos los casos que cumplieron cualquiera de los siguientes criterios: tratamiento previo para H. pylori, antecedentes de úlcera gástrica o duodenal, neoplasia de cualquier índole, ser portadores de alguna enfermedad metabólica, mujeres gestantes o en período de lactancia, tratamiento previo con antibióticos $<6$ meses, alergia a penicilinas u otros antibióticos, y antecedentes de cirugía gástrica previa. En cuanto al tamaño de muestra, se estimó que se requeriría mínimo de 50 pacientes ( 25 expuestos y 25 no expuestos) para encontrar diferencias en las tasas de erradicación $>25 \%$, y se asumió una tasa de erradicación en los no expuestos del $70 \%$, un IC exploratorio del $90 \%$ y un poder de estudio del $80 \%$. Independientemente de ello, para maximizar el poder de estudio a priori, se previó incluir a todos los pacientes expuestos que cumplieran con estos criterios de selección durante el período de estudio

\section{Terapia triple estándar para el $\boldsymbol{H}$. pylori}

Todos los pacientes infectados por el $H$. pylori recibieron la TTE de forma gratuita, la cual fue proporcionada por el hospital del Seguro Social (EsSalud) de Tarapoto. La TTE es considerada como la primera línea de tratamiento contra el H. pylori (8), y consiste en la administración por vía oral de amoxicilina ( $1 \mathrm{~g}$ dos veces al día), claritromicina ( $500 \mathrm{mg}$ dos veces al día) y omeprazol ( $20 \mathrm{mg}$ dos veces al día) por 14 días.

\section{Terapia triple estándar en adyuvancia con vitaminas C y E}

El uso de una forma adyuvante de las vitaminas $\mathrm{C}$ y $\mathrm{E}$ junto a la TTE es producto del surgimiento de nuevas y potenciales alternativas terapéuticas contra el $\mathrm{H}$. pylori. Esta terapia consiste en la administración de la vitamina C (500 mg dos veces al día) y E (200 UI dos veces al día) 30 días después de haber finalizado la TTE (9).

\section{Recolección de datos}

En todos los casos se utilizó como fuente primaria de información las historias clínicas del hospital del Seguro Social (EsSalud) de Tarapoto, donde fueron tratados tanto los expuestos como los no expuestos. En el caso de los expuestos, todos los datos de interés fueron tomados de sus historias clínicas conforme fueron identificados por el investigador principal (Wildor Samir Cubas, WSC); mientras que en el caso de los nos expuestos, primero se procedió a la elaboración de un marco muestral con los números de historias clínicas según el orden cronológico, se tomó la muestra y, recién en una segunda instancia, se procedió al levantamiento de los datos. En ambos casos se levantaron como variables de interés la edad, el género, el ámbito laboral, la procedencia, la sintomatología principal, el tiempo de enfermedad y los hallazgos endoscópicos e histopatológicos pre y posexposición a las terapias asignadas. Todas las variables de interés del estudio fueron medidas de manera estándar, y recogidas de manera retrospectiva de las historias clínicas de cada sujeto de estudio. Para facilitar la recolección de los datos, se elaboró una lista de chequeo con rangos de valores y categorías preestablecidas a fin de garantizar un levantamiento fidedigno de los datos. Terminado dicho proceso, los datos fueron doblemente digitados, y cualquier discrepancia se resolvió revisando de nuevo las historias clínicas.

\section{Análisis de datos}

Se realizó un análisis descriptivo de las características clínico-epidemiológicas basales de la población de estudio. Para ello, se calculó la media, la desviación estándar (DE) de las variables cuantitativas, la frecuencia absoluta y la relativa de las variables cualitativas. Asimismo, con el objeto de analizar la comparabilidad de las características basales de los expuestos y no expuestos, se utilizó la prueba exacta de Fisher y la prueba $\chi^{2}$ para la comparación de proporciones. Finalmente, para la estimación de la efectividad atribuible a la terapia adyuvante con los antioxidantes (vitaminas $\mathrm{Cy}$ E), se realizó un análisis de regresión de Poisson multivariante, utilizando el método de modelos anidados para aislar dicho efecto controlado por sus características basales. En este análisis se tomó como potenciales factores de confusión la edad, el género, la adherencia al tratamiento, el tiempo de enfermedad y el número de síntomas. En todos los casos, el análisis de los datos se realizó utilizando el paquete estadístico STATA MP v13, y considerando un IC del $95 \%$.

\section{Consideraciones éticas}

El comité de ética del Hospital Nacional Docente Madre Niño "San Bartolomé", en Lima, Perú, revisó y aprobó el protocolo de este estudio. Se respetó la confidencialidad de la información, y no fue necesario el uso del consentimiento informado, porque los datos se obtuvieron de forma retrospectiva.

\section{RESULTADOS}

\section{Características de la población de estudio}

Durante el período comprendido entre julio-diciembre del 2016, se evaluó un total de 50 pacientes con infección por 
H. pylori, quienes recibieron la terapia erradicadora según su asignación metodológica, de los cuales cinco pacientes (10\%) fueron excluidos del análisis porque no completaron el tratamiento. De estos cinco, dos pertenecían al grupo de los expuestos y tres al grupo de los no expuestos. Entre los 45 pacientes analizados, no se encontraron diferencias entre los expuestos y no expuestos (23 frente a 22 ) en términos de predominio del género masculino ( $65 \%$ frente a $64 \%)$ y edad promedio ( $38 \pm 11$ frente a $36 \pm 10$ años), la cual fue de $37 \pm 11$ años (rango: 19-59 años). La mayoría de los pacientes procedía de la ciudad de Tarapoto (74\% frente a $64 \%)$, y el resto de las zonas rurales de la periferia (26\% frente a $36 \%)$; la mayoría trabajaba en el sector público (52\% frente a $63 \%$ ), los restantes eran trabajadores del sector privado (39\% frente a $31 \%$ ) o desempleados (9\% frente a $5 \%$ ). El principal motivo de consulta de los pacientes en el servicio de Gastroenterología del Hospital del Seguro Social (EsSalud) de Tarapoto fue el dolor abdominal ( $43 \%$ frente a $40 \%$ ), seguido de la regurgitación (22\% frente a $27 \%)$, pirosis ( $17 \%$ frente a $18 \%)$, hiporexia $(13 \%$ frente a $5 \%$ ) y náuseas ( $4 \%$ frente a $9 \%)$. El tiempo de presentación de las manifestaciones clínicas en los participantes fue, en la mayoría de los casos, mayor a siete días ( $74 \%$ frente a $77 \%$ ). Según los hallazgos endoscópicos encontrados previos a la terapia erradicadora, las lesiones gástricas más frecuentes en los pacientes analizados fueron gastritis antral (43\% frente a $55 \%$ ), pangastritis ( $43 \%$ frente a $27 \%)$ y metaplasia intestinal leve (13\% frente a $18 \%)$.

\section{Efectividad de las terapias erradicadoras para H. pylori}

La efectividad global de las terapias erradicadoras para $H$. pylori, expresadas como la tasa de erradicación, se estimó en un $87 \%$ (IC del $95 \%$ : $76 \%$ a $97 \%$ ); mientras que las tasas de erradicación específicas encontradas en la muestra de los expuestos y no expuestos fueron del $91 \%$ (IC del $95 \%$ : 79-100 \%) frente a $82 \%$ (IC del $95 \%$ : 64-99\%), respectivamente. Con estas tasas de efectividad se estimó la razón de tasas de incidencias de 1,12 (IC del $95 \%$ : 0,88 a 1,41).

\section{DISCUSIÓN}

La terapia adyuvante con vitaminas $\mathrm{C}$ y $\mathrm{E}$ ha sido reportada como una alternativa efectiva para incrementar la tasa de erradicación de H. pylori atribuible a la TTE. Sin embargo, aunque tal podría ser el caso de los pacientes infectados con $H$ pylori en la Amazonía peruana, los resultados de nuestro estudio no nos permiten ser concluyentes al respecto. De hecho, si bien nuestros datos sugieren un efecto favorable a la terapia adyuvante (incremento en la tasa de erradicación $=9,5 \%$; $91 \%$ frente a $82 \%$; razón de tasas de incidencia $=$
1,11; IC del $95 \%$ : 0,92 a 1,36); lo cierto es que, en nuestro estudio, tal efecto resultó estadísticamente no significativo (Figura 1). Esto podría deberse a dos razones: por un lado, que dicho efecto no exista; por otro lado, que de hecho exista, pero su magnitud sea tan pequeña como para haber sido detectada con un poder de estudio tan pequeño como el nuestro.

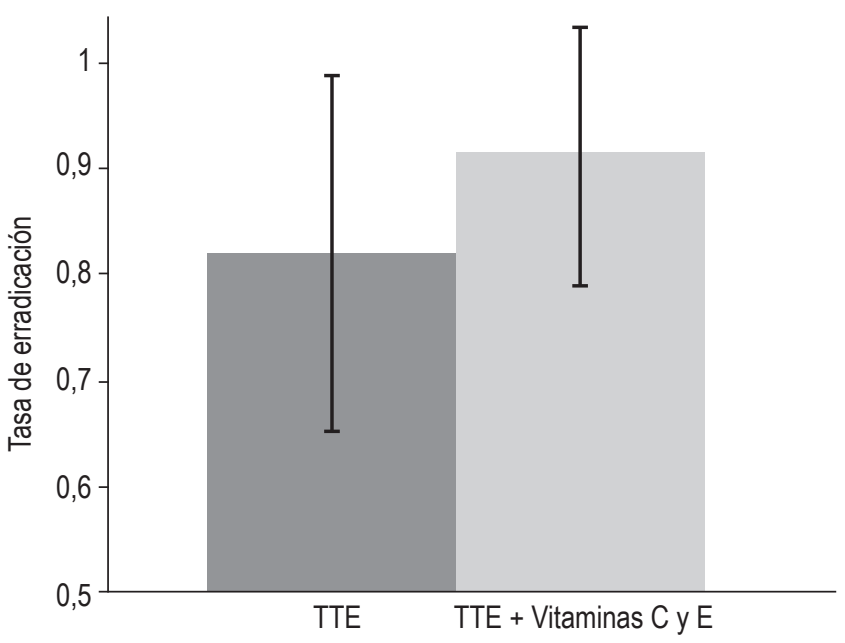

Figura 1. Efectividad atribuible al uso de las vitaminas $\mathrm{C}$ y $\mathrm{E}$ como tratamiento adyuvante a la terapia triple estándar para pacientes infectados con H. pylori, en una población de la Amazonía peruana. El tratamiento adyuvante con las vitaminas $\mathrm{C}$ y E podría ayudar a incrementar las tasas de erradicación obtenidas con TTE en los pacientes infectados con H. pylori (91\% frente a $82 \%$, razón de tasas de incidencia $=1,11$; IC del $95 \%: 0,92$ a 1,36).

De acuerdo con algunos estudios previos, las tasas de erradicación atribuibles a la TTE en adyuvancia con las vitaminas C y E pueden ser tan altas como 94 \% (9). Esta tasa de erradicación es muy similar a la encontrada en nuestro estudio (91\%) (Tabla 1). Estos valores obtenidos podrían explicarse debido a que la población estudiada probablemente contaba con algún nivel de deficiencia de su capacidad antioxidante, debido que este escenario se evidenció en un estudio experimental llevado a cabo en Asia, donde la muestra infectada por $H$. pylori, además, presentó bajos niveles de capacidad antioxidante, y que después de la administración de la terapia erradicadora en adyuvancia con vitaminas $\mathrm{C}$ y $\mathrm{E}$ se obtuvieron tasas de erradicación superiores a la terapia estándar (16). Posteriormente, estudios reportaron que individuos con bajos niveles de antioxidantes estaban relacionados con la persistencia y una mayor virulencia por $H$. pylori, y que además la mejora del mismo afectaría la supervivencia de dicha bacteria $(22,23)$. Otro factor involucrado en la patogenia por $\mathrm{H}$. pylori es su relación directa con la anemia ferropénica en los individuos infectados, debido a que existe una mala absorción del hierro en presencia de bajas concentraciones de antioxidantes, 
como es el caso de la vitamina $\mathrm{C}$ producto de la infección por $H$. pylori $(17,21)$. De tal manera que esto podría sumarse a la problemática de un país sudamericano como el Perú, cuyos últimos reportes de prevalencia de infección por $H$. pylori y anemia superan el $60 \%$ y $40 \%$, respectivamente $(19,24)$;y en regiones de la Amazonía peruana, incluyendo el departamento de San Martín, los valores de la anemia oscilan alrededor del $24 \%$ (20).

Tabla 1. Tasa de erradicación de H. Pylori según la exposición a la terapia con vitamina $\mathrm{C}$ y $\mathrm{E}$

\begin{tabular}{ccccc}
\hline \multirow{2}{*}{ Variables } & \multicolumn{2}{c}{ Expuestos } & \multicolumn{2}{c}{ No expuestos } \\
\cline { 2 - 5 } & $\mathbf{n}$ & $\%$ & $\mathbf{n}$ & $\%$ \\
\hline Erradicación & & & & \\
Sí & 21 & 91 & 18 & 82 \\
No & 2 & 9 & 4 & 18 \\
Total & 23 & 100 & 22 & 100 \\
\hline
\end{tabular}

Tomada de: historias clínicas de pacientes infectados con $H$. pylori del Servicio de Gastroenterología del Hospital del Seguro Social (EsSalud) de Tarapoto, entre los meses de julio a diciembre del 2016.

Los hallazgos encontrados en nuestro trabajo nos indican que la TTE en adyuvancia con las vitaminas $\mathrm{C}$ y $\mathrm{E}$ cuenta con resultados terapéuticos superiores frente a la TTE en monoterapia ( $91 \%$ frente a $82 \%$ ) y, teniendo en cuenta la estrecha relación que guardan la infección por $H$. pylori, la anemia y los bajos niveles de antioxidantes, podemos inferir que la suplementación con las vitaminas $\mathrm{C}$ y $\mathrm{E}$ en las terapias erradicadoras podrían haber mejorado indirectamente los niveles de antioxidantes en los sujetos de estudio, lo cual contribuye de alguna forma al éxito de las tasas de erradicación de H. pylori por encima del estándar. A pesar de la existencia de estudios en la década pasada que plantean que las evidencias disponibles para recomendar dicha terapia carecen de hallazgos significativos (15), en la actualidad se han venido desarrollando diversos trabajos con la finalidad de demostrar la efectividad terapéutica de la terapia erradicadora en adyuvancia con las vitaminas $\mathrm{C}$ y $\mathrm{E}$ (25-27).

$\mathrm{Al}$ igual que con otros estudios, las infecciones por $\mathrm{H}$. pylori fueron observadas con mayor frecuencia en pacientes adultos $(24,28,29)$ del género masculino $(30,31)$. Sin embargo, contrario a lo esperado, se observó que la mayoría de los pacientes infectados con $H$. pylori (68\%) procedían de las zonas urbanas de Tarapoto, y los restantes de las áreas rurales (Tabla 2). Esto puede deberse a un efecto del diseño, toda vez que las infecciones por $H$. pylori son comúnmente reportadas como asociadas a malas condiciones socioeconómicas y a servicios básicos deficientes con acceso limitado a agua potable; sin embargo, en el Perú, disponer de agua potable no parece ser suficiente para pre- venir nuevas infecciones por $H$. pylori. De hecho, según un estudio reciente llevado a cabo en la ciudad de Lima, donde la mayoría de la población tiene acceso a agua potable, es común encontrar restos de material genético de $H$. pylori en el agua potable (32). Y lo que es más preocupante aún, no es raro encontrar cepas de $H$. pylori resistentes a niveles estándar de hipoclorito de sodio (cloro) (33).

Tabla 2. Características de la población

\begin{tabular}{|c|c|c|c|c|}
\hline \multirow[t]{2}{*}{ Variables } & \multicolumn{2}{|c|}{ Expuestos } & \multicolumn{2}{|c|}{ No expuestos } \\
\hline & $\mathrm{n}$ & $\%$ & $\mathrm{n}$ & $\%$ \\
\hline \multicolumn{5}{|l|}{ Sexo } \\
\hline Masculino & 15 & $65 \%$ & 14 & $64 \%$ \\
\hline Femenino & 8 & $35 \%$ & 8 & $36 \%$ \\
\hline \multicolumn{5}{|l|}{ Procedencia } \\
\hline Tarapoto & 17 & $74 \%$ & 14 & $64 \%$ \\
\hline Periferia & 6 & $26 \%$ & 8 & $36 \%$ \\
\hline \multicolumn{5}{|l|}{ Ámbito laboral } \\
\hline Público & 12 & $52 \%$ & 14 & $63 \%$ \\
\hline Privado & 9 & $39 \%$ & 7 & $32 \%$ \\
\hline Desempleado & 2 & $9 \%$ & 1 & $5 \%$ \\
\hline \multicolumn{5}{|l|}{ Motivo de consulta } \\
\hline Dolor abdominal & 10 & $43 \%$ & 9 & $41 \%$ \\
\hline Regurgitación & 5 & $22 \%$ & 6 & $27 \%$ \\
\hline Pirosis & 4 & $17 \%$ & 4 & $18 \%$ \\
\hline Hiporexia & 3 & $13 \%$ & 1 & $5 \%$ \\
\hline Náuseas & 1 & $4 \%$ & 2 & $9 \%$ \\
\hline \multicolumn{5}{|l|}{ Tiempo de enfermedad } \\
\hline$>7$ días & 17 & $74 \%$ & 17 & $77 \%$ \\
\hline$<7$ días & 6 & $26 \%$ & 5 & $23 \%$ \\
\hline \multicolumn{5}{|l|}{ Histopatología inicial } \\
\hline Gastritis antral & 10 & $43 \%$ & 12 & $55 \%$ \\
\hline Pangastritis & 10 & $43 \%$ & 6 & $27 \%$ \\
\hline Metaplasia intestinal & 3 & $14 \%$ & 4 & $18 \%$ \\
\hline
\end{tabular}

Tomada de: historias clínicas de pacientes infectados con $H$. pylori del Servicio de Gastroenterología del Hospital del Seguro Social (EsSalud) de Tarapoto, entre los meses de julio a diciembre del 2016.

En conclusión, se puede decir que el tratamiento adyuvante con vitaminas $\mathrm{C}$ y $\mathrm{E}$ podría ayudar a incrementar la efectividad atribuible a la TTE para H. pylori en los pacientes de la Amazonía peruana; sin embargo, para demostrar ello se recomienda realizar más investigaciones de tipo experimental con la finalidad de obtener una mayor evidencia terapéutica. 


\section{Contribuciones de autoría}

WSC, RRC, HAR y AMQ participaron en el diseño del estudio, la interpretación de los resultados y la redacción del manuscrito; además, WSC, RRC y HAR participaron en la recolección de datos; y AMQ participó en el análisis de los datos.

\section{Conflicto de intereses}

Los autores declaran no tener conflictos de intereses en la ejecución y publicación de este artículo.

\section{REFERENCIAS}

1. Hooi JKY, Lai WY, Ng WK, Suen MMY, Underwood FE, Tanyingoh D, et al. Global Prevalence of Helicobacter pylori Infection: Systematic Review and Meta-Analysis. Gastroenterology. 2017 Aug;153(2):420-429. doi: 10.1053/j.gastro.2017.04.022.

2. Zamani M, Ebrahimtabar F, Zamani V, Miller WH, Alizadeh-Navaei R, Shokri-Shirvani J, et al. Systematic review with meta-analysis: the worldwide prevalence of Helicobacter pylori infection. Aliment Pharmacol Ther. 2018 Apr;47(7):868-876. doi: 10.1111/apt.14561.

3. Suzuki H. Helicobacter pylori-Associated Upper Gastrointestinal Symptoms: FD or HpD? Dig Dis Sci. 2017 Jun;62(6):1391-1393. doi: 10.1007/s10620-017-4556-4.

4. Schistosomes, liver flukes and Helicobacter pylori. IARC Working Group on the Evaluation of Carcinogenic Risks to Humans. Lyon, 7-14 June 1994. IARC Monogr Eval Carcinog Risks Hum. 1994;61:1-241.

5. Fallone CA, Chiba N, van Zanten SV, Fischbach L, Gisbert JP, Hunt RH, et al. The Toronto Consensus for the Treatment of Helicobacter pylori Infection in Adults. Gastroenterology. 2016 Jul;151(1):51-69.e14. doi: 10.1053/j.gastro.2016.04.006.

6. Gisbert JP, Calvet X. Review article: the effectiveness of standard triple therapy for Helicobacter pylori has not changed over the last decade, but it is not good enough. Aliment Pharmacol Ther. 2011 Dec;34(11-12):1255-68. doi: 10.1111/j.1365-2036.2011.04887.x.

7. Novoa Reyes I, Caravedo Martínez M, Huerta-Mercado TJ, De los Ríos Senmache R, Pinto Valdivia J, Bussalleu Rivera A. Recurrencia de la infección gástrica con Helicobacter pylori en adultos peruanos con distrés postprandial dos años después de la erradicación exitosa. Rev Gastroenterol del Perú. 2014;34(1):15-21.

8. Malfertheiner P, Megraud F, O’Morain CA, Gisbert JP, Kuipers EJ, Axon AT, et al. Management of Helicobacter pylori infection-the Maastricht V/Florence Consensus Report. Gut. 2017 Jan;66(1):6-30. doi: 10.1136/ gutjnl-2016-312288.

9. Sezikli M, Cetinkaya ZA, Sezikli H, Güzelbulut F, Tiftikçi A, Ince AT, et al. Oxidative stress in Helicobacter pylori infec- tion: does supplementation with vitamins $\mathrm{C}$ and $\mathrm{E}$ increase the eradication rate? Helicobacter. 2009 Aug;14(4):280-5. doi: 10.1111/j.1523-5378.2009.00686.x.

10. Zhang ZW, Abdullahi M, Farthing MJ. Effect of physiological concentrations of vitamin $\mathrm{C}$ on gastric cancer cells and Helicobacter pylori. Gut. 2002 Feb;50(2):165-9. doi: 10.1136/gut.50.2.165.

11. Zhang ZW, Farthing MJ. The roles of vitamin $\mathrm{C}$ in Helicobacter pylori associated gastric carcinogenesis. Chin J Dig Dis. 2005;6(2):53-8. doi: 10.1111/j.14439573.2005.00194.x.

12. Traber MG, Stevens JF. Vitamins C and E: beneficial effects from a mechanistic perspective. Free Radic Biol Med. 2011 Sep 1;51(5):1000-13. doi: 10.1016/j.freeradbiomed.2011.05.017.

13. Sugimoto N, Yoshida N, Nakamura Y, Ichikawa H, Naito Y, Okanoue $\mathrm{T}$, et al. Influence of vitamin $\mathrm{E}$ on gastric mucosal injury induced by Helicobacter pylori infection. Biofactors. 2006;28(1):9-19. doi: 10.1002/biof.5520280102.

14. Everett SM, Drake IM, White KL, Mapstone NP, Chalmers DM, Schorah CJ, et al. Antioxidant vitamin supplements do not reduce reactive oxygen species activity in Helicobacter pylori gastritis in the short term. Br J Nutr. 2002 Jan;87(1):311. doi: 10.1079/BJN2001477.

15. Li G, Li L, Yu C, Chen L. Effect of vitamins $C$ and $E$ supplementation on Helicobacter pylori eradication: a meta-analysis. Br J Nutr. 2011 Dec;106(11):1632-7. doi: 10.1017/ S0007114511003813.

16. Sezikli M, Cetinkaya ZA, Güzelbulut F, Sezikli H, Özkara S, Coşgun $S$, et al. Efficacy of vitamins supplementation to therapy on Helicobacter pylori eradication in patients with low antioxidant capacity. Clin Res Hepatol Gastroenterol. 2011 Nov;35(11):745-9. doi: 10.1016/j.clinre.2011.07.001.

17. Hudak L, Jaraisy A, Haj S, Muhsen K. An updated systematic review and meta-analysis on the association between Helicobacter pylori infection and iron deficiency anemia. Helicobacter. 2017 Feb;22(1). doi: 10.1111/hel.12330.

18. Franceschi F, Annalisa T, Teresa DR, Giovanna D, Ianiro G, Franco S, et al. Role of Helicobacter pylori infection on nutrition and metabolism. World J Gastroenterol. 2014 Sep 28;20(36):12809-17. doi: 10.3748/wig.v20.i36.12809.

19. Alcázar L. Impacto económico de la anemia en el Perú. Lima: GRADE; Acción contra el Hambre; 2012. p. 19-24.

20. Tarqui-Mamani C, Sanchez-Abanto J, Alvarez-Dongo D, Espinoza-Oriundo P, Jordan-Lechuga T. Prevalencia de anemia y factores asociados en adultos mayores peruanos. Rev Peru Med Exp Salud Pública. 2015;32(4):687-92. doi: 10.17843/rpmesp.2015.324.1759

21. Lane DJ, Jansson PJ, Richardson DR. Bonnie and Clyde: Vitamin $\mathrm{C}$ and iron are partners in crime in iron deficiency anaemia and its potential role in the elderly. Aging (Albany NY). 2016 May;8(5):1150-2. doi: 10.18632/aging.100966.

22. Sezikli M, Çetinkaya ZA, Güzelbulut F, Çimen B, Özcan Ö, Özkara S, et al. Effects of alpha tocopherol and ascorbic acid on Helicobacter pylori colonization and the severity of gas- 
tric inflammation. Helicobacter. 2012 Apr;17(2):127-32. doi: 10.1111/j.1523-5378.2011.00925.x.

23. Hagag AA, Amin SM, El-Fiky RB, El-Sayad ME. Study of Serum Levels of Some Oxidative Stress Markers in Children with Helicobacter pylori Infection. Infect Disord Drug Targets. 2018;18(1):52-59. doi: 10.2174/1871526517666 170102115116.

24. Pareja Cruz A, Navarrete Mejía PJ, Parodi García JF. Seroprevalencia de infección por Helicobacter pylori en población adulta de Lima, Perú 2017. Horizonte Médico. 2017;17(2):55-8. doi: 10.24265/horizmed.2017.v17n2.08.

25. Tümgör G, Baran M, Çakır M, Yüksekkaya HA, Aydoğdu S. Comparison of standard and standard plus vitamin $\mathrm{E}$ therapy for Helicobacter pylori eradications in children. Turk J Gastroenterol. 2014 Dec;25 Suppl 1:99-103. doi: 10.5152/ tjg.2014.5592.

26. Demirci H, Uygun İlikhan S, Öztürk K, Üstündağ Y, Kurt Ö, Bilici $M$, et al. Influence of vitamin $C$ and $E$ supplementation on the eradication rates of triple and quadruple eradication regimens for Helicobacter pylori infection. Turk J Gastroenterol. 2015 Nov;26(6):456-60. doi: 10.5152/ tjg.2015.0233.

27. Sezikli M, Çetinkaya ZA, Güzelbulut F, Yeşil A, Coşgun S, Kurdaş OÖ. Supplementing vitamins $\mathrm{C}$ and $\mathrm{E}$ to standard triple therapy for the eradication of Helicobacter pylori. J Clin Pharm Ther. 2012 Jun;37(3):282-5. doi: 10.1111/j.13652710.2011.01286.x.

28. Bui D, Brown HE, Harris RB, Oren E. Serologic Evidence for Fecal-Oral Transmission of Helicobacter pylori. Am
J Trop Med Hyg. 2016 Jan;94(1):82-8. doi: 10.4269/ ajtmh.15-0297.

29. Castillo Contreras O, Maguiña Quispe J, Benites Goñi H, Chacaltana Mendoza A, Guzmán Calderón E, Dávalos Moscol M, et al. Prevalencia de Helicobacter pylori en pacientes sintomáticos de consulta externa de la Red Rebagliati (EsSalud), Lima, Perú, en el período 2010-2013. Rev Gastroenterol Perú. 2016;36(1):49-55.

30. Wex T, Venerito M, Kreutzer J, Götze T, Kandulski A, Malfertheiner P. Serological prevalence of Helicobacter pylori infection in Saxony-Anhalt, Germany, in 2010. Clin Vaccine Immunol. 2011 Dec;18(12):2109-12. doi: 10.1128/CVI.05308-11.

31. Zhang M, Zhou YZ, Li XY, Tang Z, Zhu HM, Yang Y, Chhetri JK. Seroepidemiology of Helicobacter pylori infection in elderly people in the Beijing region, China. World J Gastroenterol. 2014 Apr 7;20(13):3635-9. doi: 10.3748/ wjg.v20.i13.3635.

32. Boehnke KF, Brewster RK, Sánchez BN, Valdivieso M, Bussalleu A, Guevara M, et al. An assessment of drinking water contamination with Helicobacter pylori in Lima, Peru. Helicobacter. 2018 Apr;23(2):e12462. doi: 10.1111/ hel.12462.

33. Ramírez A, Chinga E, Mendoza D. Variación de la prevalencia del H. Pylori y su relación con los niveles de cloro en el agua de la Atarjea, Lima, Perú: Período 1985-2002. Rev Gastroenterol Perú. 2004;24(3):223-9. 